\title{
Kinematical data on early-type galaxies. V. ${ }^{\star, \star \star}$
}

\author{
F. Simien and Ph. Prugniel \\ CRAL-Observatoire de Lyon (CNRS: UMR 142), 69561 St-Genis-Laval Cedex, France
}

Received April 3; accepted May 23, 2000

\begin{abstract}
We present kinematical data for a sample of 26 galaxies. Rotation curves and velocity-dispersion profiles are determined for all objects. This is our fifth paper in a series devoted to the presentation of data on elliptical and S0 galaxies, derived from long-slit absorption spectroscopy; the series now gathers 119 galaxies with homogeneous data.
\end{abstract}

Key words: galaxies: elliptical \& lenticular, cD — galaxies: kinematics and dynamics - galaxies: fundamental parameters — galaxies: general

\section{Introduction}

We have presented kinematical measurements from absorption spectroscopy on early-type galaxies (Simien \& Prugniel 1997a, 1997b, 1997c, 1998: hereafter Papers I to IV, respectively); these data are intended to contribute to the study of several structural and evolutionary issues. As part of our ongoing effort to get reliable velocity dispersions and rotation curves on a statistically significant sample of objects, we presently report on observations on a fifth list of targets.

This work follows closely the technique already described in full detail in Paper I, for both observation and reduction, and only a minimum of explanations will be given here.

Send offprint requests to: F. Simien,

e-mail: simien@obs.univ-lyon1.fr

* Based on observations collected at the Observatoire de Haute-Provence.

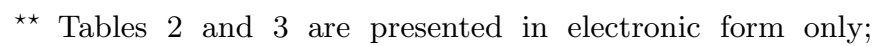
Tables 1 through 3 are available from the CDS, Strasbourg, via anonymous ftp to cdsarc.u-strasbg.fr (130.79.128.5) or via http://cdsweb.u-strasbg.fr/Abstract.html

\section{Sample and observations}

Our present sample consists of 26 early-type galaxies, with 13 ellipticals and 13 S0s. There are five objects in common with the preceding papers of the series; these duplicate measurements have been included in the present work for comparison purposes. Relevant catalog elements are presented in the first 10 columns of Table 1. The Es have ellipticities corresponding to classes between $\simeq \mathrm{E} 1$ and $\simeq \mathrm{E} 3$, and the S0s are moderately to highly flattened. The distances are between $\simeq 10$ to $\simeq 80 \mathrm{Mpc}$ (for $H_{0}=75 \mathrm{~km} \mathrm{~s}^{-1} \mathrm{Mpc}^{-1}$ ). The absolute magnitudes of these objects are in the range $-22.0<M_{B}<-18.0$.

The observations have been secured at the 1.93-m telescope of the Observatoire de Haute-Provence, equipped with the CARELEC long-slit spectrograph. The selected setup provided a spectral range of $\simeq 900 \AA$ centered on $\lambda=5200 \AA$ with a FWHM resolution of $\simeq 3.2 \AA$ corresponding to an intrumental velocity dispersion of $\simeq 82 \mathrm{~km} \mathrm{~s}^{-1}$. The slit width, projected onto the plane of the sky, was $2.2^{\prime \prime}$.

In December 1997, an observing run totalling six clear nights allowed us to collect one or two spectra on each object, along the major axis or an axis close to it. For NGC 2314 and NGC 2634, additional spectra were secured along the minor axis.

The atmospheric conditions were variable, with a seeing disk between $2^{\prime \prime}$ and $3^{\prime \prime}$ (FWHM) for most objects, but more than $4^{\prime \prime}$ for three of them. These variations are an additional justification of the duplicate measurements for the same object. The log of the observations is given in Table 2, which is proposed in electronic form only.

\section{Data reduction}

As in Papers I to IV, standard pre-processing was applied to the raw data, up to the rebinning in wavelength. The galaxy centers $(r=0)$ were determined by a Gaussian fitting to a limited range $\left(\simeq 12^{\prime \prime}\right)$ around the intensity peak. In the outer regions, cosmic-ray hits were removed 
Table 1. Catalog elements and kinematical results

\begin{tabular}{|c|c|c|c|c|c|c|c|c|c|c|c|c|c|}
\hline Object & Type & $\alpha_{1950}$ & $\delta_{1950}$ & $B_{\mathrm{T}}$ & $-M_{B}$ & $r_{\mathrm{e}}$ & $\epsilon$ & $\mathrm{PA}$ & ref. & $c z$ & $\sigma_{0}$ & $V_{\max }$ & $\overline{r_{\max }}$ \\
\hline (1) & $(2)$ & (3) & (4) & (5) & (6) & (7) & $(8)$ & $(9)$ & (10) & (11) & $(12)$ & (13) & $\overline{(14)}$ \\
\hline IC 0464 & -3 & 70714.6 & $\begin{array}{llll}50 & 13 & 09\end{array}$ & 4.94 & 20.02 & 7.2 & .45 & 50 & 3 & $4818 \pm 19$ & $11 \pm 08$ & $180 \pm 09$ & 27 \\
\hline NGC 0194 & -5 & 003644.0 & 024542 & 3.09 & .13 & 19.7 & .05 & 38 & 1 & $5228 \pm 11$ & $32 \pm 08$ & $3 \pm 13$ & 20 \\
\hline NGC 0474 & -2 & 011731.7 & -030916 & 12.31 & 0.06 & 34.5 & .19 & 21 & 1 & $2317 \pm 08$ & $163 \pm 05$ & $0 \pm 06$ & 30 \\
\hline NGC 0524 & -1 & 012210.1 & +091645 & 11.46 & 21.27 & 36.2 & .01 & 38 & 3 & $2379 \pm 15$ & $257 \pm 08$ & $124 \pm 07$ & 38 \\
\hline NGC 0680 & -5 & $\begin{array}{lll}01 & 47.4\end{array}$ & +214322 & 12.66 & 20.54 & 19.2 & .10 & 158 & 1 & $2867 \pm 11$ & $214 \pm 06$ & $108 \pm 07$ & 34 \\
\hline NGC 0731 & -5 & 015227.6 & -091520 & 13.09 & 20.68 & 14.1 & .06 & 150 & 3 & $3832 \pm 09$ & $171 \pm 05$ & $6 \pm 06$ & 20 \\
\hline NGC & -1 & $\begin{array}{lll}02 & 11 & 10.7\end{array}$ & +273839 & 13.53 & 6.82 & 13.8 & .57 & 66 & 2 & $5 \pm 09$ & $0 \pm 16$ & $3 \pm 10$ & 20 \\
\hline NGC 1550 & -5 & 041702.0 & +021725 & 13.22 & 20.85 & 13.9 & .08 & 33 & 1 & $3764 \pm 17$ & $326 \pm 14$ & $\geq 30 \pm 20$ & 18 \\
\hline NGC 1573 & -5 & 042903.2 & +730933 & 12.82 & 21.63 & 32.2 & .31 & 30 & 3 & $4225 \pm 20$ & $304 \pm 18$ & $50 \pm 12$ & 26 \\
\hline NGC 1601 & -2 & 042913.6 & -050956 & 14.73 & 19.47 & 9.4 & .38 & 95 & 2 & \pm 21 & $191 \pm 10$ & $193 \pm 20$ & 19 \\
\hline NGC 1603 & -2 & 042921.7 & -051159 & 4.54 & 9.67 & 5.4 & .32 & 40 & 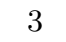 & $4972 \pm 14$ & \pm 07 & \pm 08 & 17 \\
\hline NGC 1684 & -5 & 045000.9 & -031118 & 12.82 & 1.38 & 25.9 & .33 & 90 & 3 & $4426 \pm 17$ & $301 \pm 11$ & $110 \pm 16$ & 14 \\
\hline NGC 2314 & -5 & 070353.7 & +752428 & 13.16 & 20.78 & 11.9 & .12 & 1 & 3 & \pm 20 & $293 \pm 09$ & $164 \pm 18$ & 20 \\
\hline NGC 2340 & -5 & 070720.4 & +501524 & 12.86 & 22.11 & 44.0 & .33 & 80 & 3 & $5902 \pm 11$ & \pm 09 & $\leq 17 \pm 12$ & 26 \\
\hline C 2493 & -2 & 075701.5 & +395805 & 12.93 & 21.10 & 24.4 & .00 & 35 & 0 & \pm 15 & $233 \pm 08$ & $75 \pm 09$ & 30 \\
\hline NGC 2577 & -5 & 081946.6 & +224250 & 12.38 & 20.84 & .... & .38 & 105 & 2 & \pm 11 & $230 \pm 08$ & $204 \pm 08$ & 32 \\
\hline NGC 2634 & -5 & 084256.0 & +740906 & 12.88 & 19.83 & 26.0 & .00 & 0 & 3 & $2250 \pm 09$ & $191 \pm 06$ & $65 \pm 16$ & 34 \\
\hline NGC 2636 & -5 & 084258.9 & +735115 & 14.57 & 18.14 & 7.3 & .00 & 90 & 3 & $2072 \pm 09$ & $59 \pm 06$ & $28 \pm 09$ & 12 \\
\hline NGC 2699 & -5 & 085317.6 & -025608 & 13.18 & 19.27 & 13.7 & .06 & 45 & 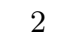 & $1846 \pm 09$ & $144 \pm 04$ & $83 \pm 08$ & 19 \\
\hline NGC 2962 & -1 & 093816.7 & +052339 & 12.64 & 20.05 & 39.5 & .25 & 175 & 3 & $1955 \pm 09$ & $182 \pm 06$ & $175 \pm 09$ & 39 \\
\hline NGC 2970 & -5 & 094032.8 & +321220 & 14.42 & 17.78 & 12.2 & .22 & 75 & 3 & $1618 \pm 08$ & $43 \pm 16$ & $\leq 22 \pm 09$ & 11 \\
\hline NGC 3619 & 0 & 111628.6 & +580159 & 12.57 & 19.88 & 23.2 & .22 & 35 & 3 & $1553 \pm 09$ & $168 \pm 05$ & $65 \pm 07$ & 26 \\
\hline NGC 3630 & -1 & 111742.1 & +031422 & 12.88 & 19.39 & 11.7 & .59 & 42 & 3 & $1485 \pm 09$ & $176 \pm 06$ & $168 \pm 08$ & 28 \\
\hline NGC 7280 & -1 & 222401.6 & +155339 & 12.93 & 19.25 & 18.6 & .30 & 75 & 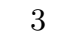 & $1832 \pm 11$ & $107 \pm 06$ & $83 \pm 09$ & 18 \\
\hline NGC 7612 & -1 & $2317 \quad 12.2$ & +081808 & 13.70 & 19.82 & 14.3 & .50 & 0 & 3 & $3213 \pm 11$ & $182 \pm 05$ & $214 \pm 10$ & 28 \\
\hline NGC 7617 & -1 & 231737.1 & +075330 & 14.92 & 18.66 & 8.1 & .28 & 29 & 3 & $4094 \pm 17$ & $108 \pm 13$ & $159 \pm 18$ & 12 \\
\hline
\end{tabular}

Notes. Col. (2): morphological type from the LEDA database (status: January 17, 2000), coded with -5 for all ellipticals; Cols. (3), (4): coordinates, from Prugniel \& Héraudeau (1998: hereafter PH98); Col. (5): $B_{\mathrm{T}}$, integrated blue magnitude, corrected for Galactic extinction according the Burstein \& Heiles (1982) model, and for $k$ term (from PH98, except for NGC 2577 which is from LEDA); Col. (6): $M_{B}$, absolute $B$ magnitude; the distance moduli are computed using the flowsmoothed velocity from Hypercat (Prugniel et al. 1999) corrected for the particular streamings as in Prugniel \& Simien (1997), and using $H_{0}=75 \mathrm{~km} \mathrm{~s}^{-1} \mathrm{Mpc}^{-1}$; except for NGC 2577: $M_{B}$ from LEDA; Col. (7): $r_{\mathrm{e}}$, effective radius in arcsec, corrected for cosmological dimming effect, from PH98; Col. (8): $\epsilon$, ellipticity, derived from parameter $R_{25}$ in LEDA; Col. (9): PA, position angle of major axis, in degrees (North through East); Col. (10): reference for PA, $1=$ Djorgovski $(1985), 2=$ LEDA, $3=$ this work (value determined on an image from the Digitized Sky Survey); Col. (11): $c z$, heliocentric radial velocity, in km $\mathrm{s}^{-1}$; Col. (12): $\sigma_{0}$, central velocity dispersion, in $\mathrm{km} \mathrm{s}^{-1}$; Col. (13): $V_{\max }$, maximum rotation velocity, in $\mathrm{km} \mathrm{s}^{-1}$ (the $\geq$ sign indicates that the rotation is still rising at the outermost point, or that the PA of the slit was inclined with respect to the major axis); Col. (14): $r_{\max }$, the radius defining the range $\left(-r_{\max }, r_{\max }\right)$ in which $V_{\max }$ was measured, in arcsec.

with a median filter, and adjacent lines were combined with a variable weighting function (a Gaussian continuously wider faintward). A Fourier-Fitting technique determined the central velocity dispersion $\sigma_{0}$ and, when possible, the radial profile $\sigma(r)$ of the dispersion, together with the projected rotation curve $V(r)$ along the major axis. A two-pass mode (described in Paper I) allowed to remove cosmics on the inner lines, where the spatial resolution must be preserved. We adopted as $\sigma_{0}$ the dispersion measured on the line at $r=0$, or an interpolation between the values on the two lines bracketting the position $r=0$; similarly, the systemic velocity corresponds to $r=0$. Whenever possible, we have determined the maximum rotation velocity $V_{\max }$, as the mean of representative values along opposite semi-axes.

\section{Presentation of the results}

Determinations of the heliocentric radial velocity $c z$, of $\sigma_{0}$, and of $V_{\max }$ (together with the radius $r_{\max }$ of the measurement range) are listed in the last columns of Table 1. The $V(r)$ and $\sigma(r)$ profiles are presented in Fig. 1, and also in Table 3, which is proposed in electronic form only. Following Papers III and IV, we have adopted a convention for the actual position angle PA of the spectrograph slit (as listed in Table 2): for $0<\mathrm{PA}<180^{\circ}$, $r<0$ corresponds to the eastern side of the galaxy, for $180<\mathrm{PA}<360^{\circ}, r<0$ corresponds to the western side, and for $\mathrm{PA}=0^{\circ}, r<0$ is to the North. Tables 1 through 3 are available from the CDS. 

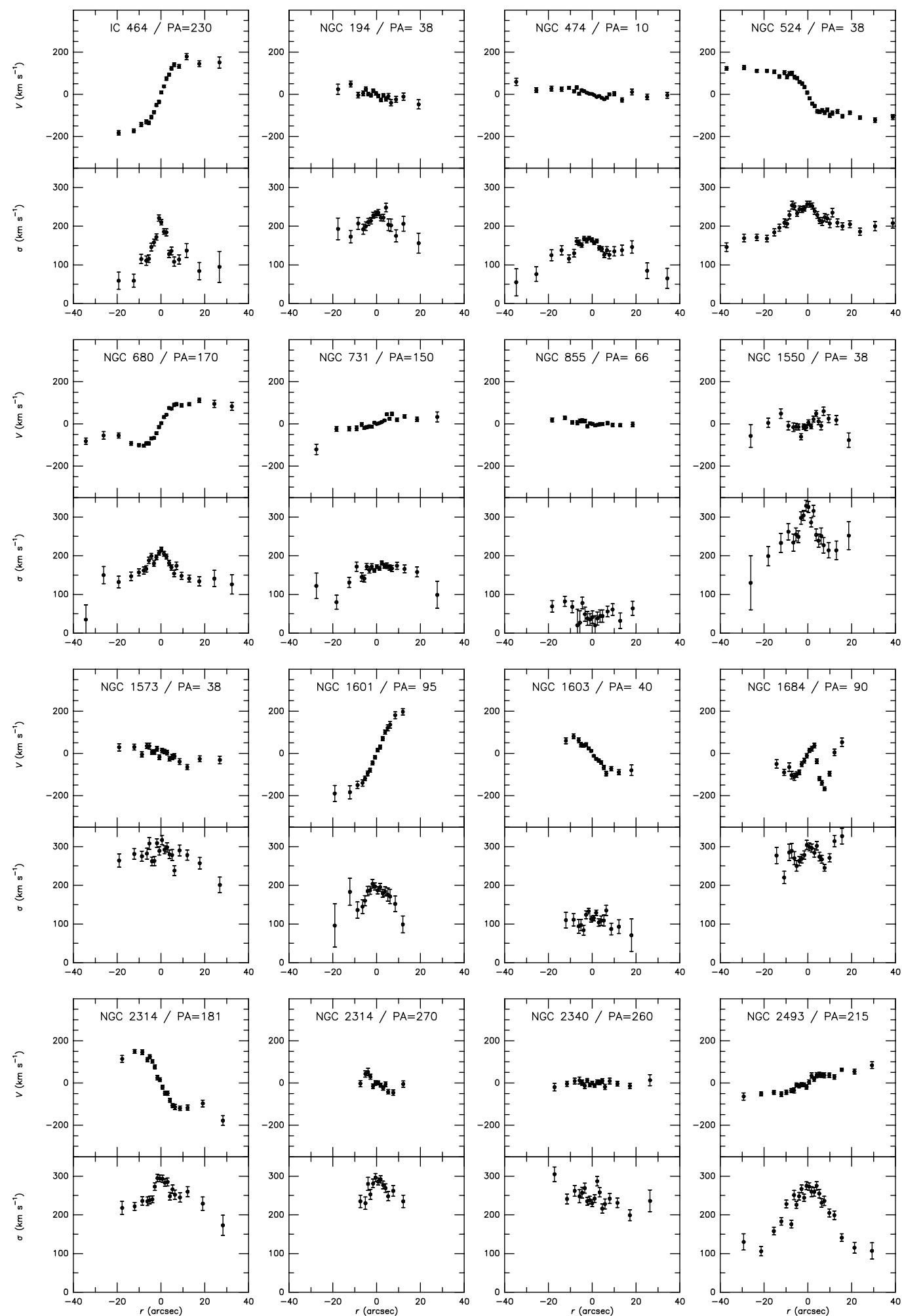

Fig. 1. Profiles of rotation velocities and velocity dispersions 

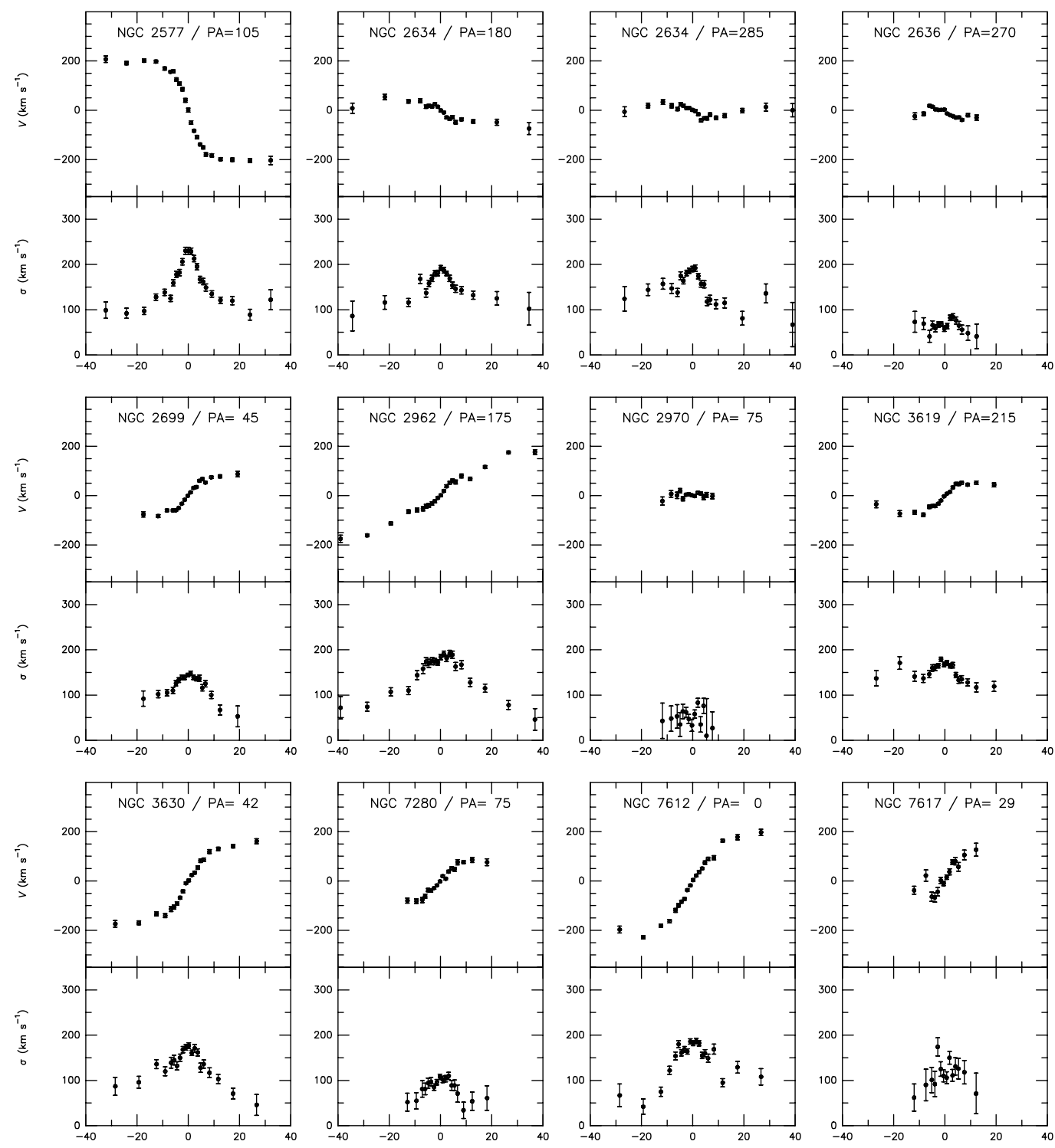

Fig. 1. continued

Our results are summarized as follows:

- We have measured the central velocity dispersion $\sigma_{0}$ for 26 early-type galaxies: these values were still unavailable for five objects. We have also determined the $\sigma(r)$ and $V(r)$ profiles along the major axis and, for most galaxies, we have been able to measure the maximum rotation velocity $V_{\max }$ : this parameter was still unavailable for 21 of these objects;

- We note several peculiar features. In NGC 1550, the rotation curve is unexplainably distorted; in NGC 1684, a secondary photometric peak is present at $\simeq 6^{\prime \prime}$ West of the nucleus, where the kinematic profiles are distorted; in NGC 2634, the amount of rotation at $\mathrm{PA}=285^{\circ}$, i.e. close to the minor axis, is similar to that along the major axis.
The data presented here, together whith those of the preceding papers of the series, are available in the HYPERCAT database (Prugniel et al. 1999), at http: //www-obs . univ-lyon1.fr/hypercat/.

Acknowledgements. We are endebted to the telescope operators at the Observatoire de Haute-Provence for their help in collecting the data. We thank the referee, Giovanni Busarello, for his careful reading of the first version of the manuscript. We have made use of the Lyon-Meudon Extragalactic Database operated by the LEDA team. We have also made use of the ESO/ST-ECF first Digitized Sky Survey (DSS). The DSS was produced at the Space Telescope Science Institute under U.S. Government grant NAG W-2166; its images are based on photographic data from the Oschin Schmidt Telescope on Palomar Mountain and the UK Schmidt Telescope. The plates were processed into the present form with the permission 
of these institutions. The National Geographic Society Palomar Observatory Sky Atlas was made by the California Institute of Technology with grants from the National Geographic Society. The Oschin Schmidt Telescope is operated by the California Institute of Technology and Palomar Observatory. The UK Schmidt Telescope was operated by the Royal Observatory Edinburgh, with funding from the UK Science and Engineering Research Council (later the UK Particle Physics and Astronomy Research Council), until 1988 June, and thereafter by the Anglo-Australian Observatory; plates of the Sky Atlas Equatorial Extension are from the UK Schmidt.

\section{References}

Burstein D., Heiles C., 1982, AJ 87, 1165

Djorgovski S., 1985, Ph.D. Thesis. Univ. of California, Berkeley Prugniel Ph., Golev V., Maubon G., 1999, A\&A 346L, 25 Prugniel Ph., Héraudeau Ph., 1998, A\&AS 128, 299 (PH98) Prugniel Ph., Simien F., 1997, A\&A 321, 111 (PS97)

Simien F., Prugniel Ph., 1997a, A\&AS 122, 521 (Paper I)

Simien F., Prugniel Ph., 1997b, A\&AS 126, 15 (Paper II)

Simien F., Prugniel Ph., 1997c, A\&AS 126, 519 (Paper III)

Simien F., Prugniel Ph., 1998, A\&AS 131, 287 (Paper IV) 